\title{
ARTICLE Ouabain impairs cancer metabolism and activates AMPK-Src signaling pathway in human cancer cell lines
}

\author{
Jia-jia Shen ${ }^{1}$, Yue-chen Zhan ${ }^{1}$, Hui-ying $\mathrm{Li}^{1}$ and Zhen Wang ${ }^{1}$
}

\begin{abstract}
In addition to the well-known cardiotonic effects, cardiac glycosides (CGs) produce potent anticancer effects with various molecular mechanisms. We previously show that ouabain induces autophagic cell death in human lung cancer cells by regulating AMPKmediated mTOR and Src-mediated ERK1/2 signaling pathways. However, whether and how AMPK and Src signaling interacts in ouabain-treated cancer cells remains unclear. Given the pivotal role of AMPK in metabolism, whether ouabain affects cancer cell metabolism remains elusive. In this study we showed that treatment with ouabain $(25 \mathrm{nM})$ caused simultaneous activation of AMPK and Src signaling pathways in human lung cancer A549 cells and human breast cancer MCF7 cells. Cotreatment with AMPK inhibitor compound C or siRNA greatly abrogates ouabain-induced Src activation, whereas cotreatment with Src inhibitor PP2 has little effect on ouabain-induced AMPK activity, suggesting that AMPK served as an upstream regulator of the Src signaling pathway. On the other hand, ouabain treatment greatly depletes ATP production in A549 and MCF7 cells, and supplement of ATP (100 $\mu$ M) blocked ouabain-induced AMPK activation. We further demonstrated that ouabain greatly inhibited the mitochondrial oxidative phosphorylation (OXPHOS) in the cancer cells, and exerted differential metabolic effects on glycolysis depending on cancer cell type. Taken together, this study reveals that the altered cancer cell metabolism caused by ouabain may contribute to AMPK activation, as well as its cytotoxicity towards cancer cells.
\end{abstract}

Keywords: ouabain; AMPK; Src; cancer; oxidative phosphorylation; glycolysis

Acta Pharmacologica Sinica (2020) 41:110-118; https://doi.org/10.1038/s41401-019-0290-0

\section{INTRODUCTION}

Cardiac glycosides (CGs), including digoxin, ouabain, digitoxin, and oleandrin, are well-known $\mathrm{Na}^{+} / \mathrm{K}^{+}$-ATPase (NKA) inhibitors that have long been used for the treatment of congestive heart failure and arrhythmia. Despite their cardiotonic effects, the potent anticancer efficacy of CGs has been widely reported for many cancer types, including breast cancer, lung cancer, prostate cancer, melanoma, colon cancer, and leukemia, with some derivative compounds having entered clinical studies [1, 2].

To date, a variety of mechanisms addressing the anticancer effects of CGs have been identified, including the induction of apoptosis and cell cycle arrest, inhibition of topoisomerase II and estrogen receptor, blockage of p53 and HIF-1a protein synthesis, and the generation of reactive oxygen species, as well as the inhibition of migration and metastasis [3-6]. Interestingly, CG class compounds have been found to be immunogenic cell death inducers that promote the immunological clearance of cancer cells and increase the efficacy of anticancer drugs by modulating the immunosuppressive function of T cells [7].

Our recent study demonstrated that autophagic cell death, which contributes to the cytotoxicity of digoxin and ouabain, is induced by CG compounds in human non-small cell lung cancer cells via the regulation of both the AMP-activated protein kinase (AMPK)-mediated mammalian target of rapamycin (mTOR) signaling pathway and the Src-mediated extracellular signal-regulated kinase $1 / 2$ (ERK1/2) pathway $[6,8]$. However, given the complexity of the AMPK and Src signaling pathways in cells [9-11], whether and how AMPK and Src signaling may cross-communicate with each other following CGs treatment remains unknown. Furthermore, as AMPK plays a pivotal role in regulating cellular energy homeostasis [12], whether CGs-induced AMPK activation results from altered cancer cell metabolism is worthy of assessment.

In the present work, we found that ouabain induces the rapid activation of AMPK, which acts as an upstream regulator of the Src signaling pathway in the A549 and MCF7 cancer cell lines. Furthermore, ouabain greatly decreases mitochondrial oxidative phosphorylation (OXPHOS) but has differential effects on glycolysis in A549 and MCF7 cells. The altered cancer metabolism caused by ouabain may contribute to AMPK activation, as well as to its cancer cell cytotoxicity.

\section{MATERIALS AND METHODS}

Antibodies and reagents

The anti- $\beta$-actin (A1978) antibody, compound C (P5499), PP2 (P0042), and ouabain (O3125) were purchased from Sigma-Aldrich (Saint Louis, MO, USA). The anti-p-AMPKa (2535), anti-AMPKa (2532), anti-p-Src (2101), anti-Src (2108), anti-p-ERK1/2 (4370), antiERK1/2 (4695), anti-p-ACC (3661), anti-p-LKB1 (3482) antibodies, and horseradish peroxidase-linked secondary antibody (7074) were

${ }^{1}$ Biochemistry Department, Institute of Medicinal Biotechnology, Peking Union Medical College \& Chinese Academy of Medical Sciences, Beijing 100050, China Correspondence: Zhen Wang (wangzhen@imb.pumc.edu.cn)

These authors contributed equally: Jia-jia Shen, Yue-chen Zhan

Received: 10 January 2019 Accepted: 17 July 2019

Published online: 12 September 2019 
purchased from Cell Signaling Technology (Beverly, MA, USA). 5Aminoimidazole-4-carboxamide-1- $\beta-D$-ribofuranoside (AICAR, sc200659) was purchased from Santa Cruz Biotechnology (Santa Cruz, CA, USA). The ATPlite Luminescence Assay System (6016941) was purchased from PerkinElmer (Wolsem, MA, USA). The CycLex AMPK Kinase Assay kit (CY-1182) was purchased from MBL Company (Nagano, Japan). The XFp Cell Mito Stress Test (103010$100)$ and Glycolysis Stress Test kits (103017-100) were purchased from Agilent Technologies (Palo Ato, CA, USA). The Glucose Assay kit (glucose oxidase method, 180831) was purchased from Biosino Biotechnology and Science Inc (Beijing, China). The Lactate Acid Assay kit (A019-2) was purchased from Nanjing Jiancheng Bioengineering Institute (Nanjing, China).

siRNA transfection

siRNA duplexes against AMPK were synthesized by RiboBio Co., Ltd (Guangzhou, China) (si-AMPK \#1: sense sequence 5'-GCAGA AGTATGTAGAGCAA-3'; si-AMPK \#2: sense sequence 5'-ACACATG AATGCAAAGATA-3'). The silencing of genes using $10 \mathrm{nM}$ RNAi duplexes was performed with Lipofectamine ${ }^{T M}$ RNAiMAX Reagent (13778-150, Life Technologies, Carlsbad, CA, USA) according to the manufacturer's recommendations.

Cell culture and drug treatment

The MCF7 cell line was maintained in DMEM (HyClone, Logan, UT, USA), while the A549 cell line was maintained in Ham's F12 medium (HyClone, Logan, UT, USA); both media were supplemented with $10 \%(\mathrm{v} / \mathrm{v})$ fetal bovine serum (Life Technologies, Carlsbad, CA, USA), $100 \mathrm{U} / \mathrm{mL}$ penicillin $\mathrm{G}$ and $100 \mu \mathrm{g} / \mathrm{mL}$ streptomycin (HyClone, Logan, UT, USA). The cells were cultured in a humidified atmosphere containing $5 \% \mathrm{CO}_{2}$ at $37^{\circ} \mathrm{C}$. Unless specifically described, the concentration for ouabain treatment in this work was $25 \mathrm{nM}$. For the inhibitors, $10 \mu \mathrm{M}$ compound $\mathrm{C}$ or 10 $\mu \mathrm{M}$ PP2 was used in the cotreatment with ouabain or AICAR $(0.5$ $\mathrm{mM})$, as indicated.

Immunoblotting (IB) analysis

Cells were seeded at $3 \times 10^{5}$ cells per well in $60 \mathrm{~mm}$ Petri dishes for $24 \mathrm{~h}$ and treated as indicated for different lengths of time. The cells were washed with ice-cold PBS and lysed with lysis buffer containing a protease inhibitor cocktail plus $1 \mathrm{mM}$ PMSF. The protein concentration was determined by the Bradford assay. Protein extracts $(30-50 \mu \mathrm{g})$ were separated by SDS-PAGE as described before [6].

Semi-quantitative RT-PCR

Cells were seeded in six-well plates at $1.5 \times 10^{5}$ cells/well for $24 \mathrm{~h}$ and then treated with $25 \mathrm{nM}$ ouabain for the indicated time. RNA was extracted using TRIzol (\#15596026, Invitrogen, Carlsbad, CA, USA). CDNA was synthesized using PrimeScript RT Master Mix (\#DRR036A, TaKaRa, Shiga, Japan). The reaction conditions used for the PCR were cycles for denaturation at $94^{\circ} \mathrm{C}$ for $30 \mathrm{~s}$, annealing at $65^{\circ} \mathrm{C}$ for $30 \mathrm{~s}$, and elongation at $72{ }^{\circ} \mathrm{C}$ for $40 \mathrm{~s}$. Intensity values of the bands were determined with ImageJ software (National Institutes of Health, Bethesda, MD, USA) and normalized to the intensity of the GAPDH band. The sequences of the primers were as follows: AMPK forward primer, $5^{\prime}$-TTGAAACCT GAAAATGTCCTGCT-3' and reverse primer, 5'- GGTGAGCCACAA CTTGTTCTT-3'.

Measurement of AMPK activity

Cells were seeded in six-well plates at $1.5 \times 10^{5}$ cells/well for $24 \mathrm{~h}$ and then treated with $0.5 \mathrm{mM}$ AICAR for $30 \mathrm{~min}$ or $25 \mathrm{nM}$ ouabain for the indicated time. The relative AMPK activity was measured using a CycLex AMPK Kinase Assay kit according to the manufacturer's instructions. Briefly, the cells were lysed with lysis buffer, $10 \mu \mathrm{L}$ of the cell lysate (containing $1 \mu \mathrm{g}$ protein) was added to the microplate, and $90 \mu \mathrm{L}$ of kinase reaction buffer (50 $\mathrm{MM}$ ATP) was added to each well.
After incubation at $30^{\circ} \mathrm{C}$ for $30 \mathrm{~min}$, the reactions were washed three times with wash buffer, $100 \mu \mathrm{L}$ of anti-phospho-mouse IRS-1 S789 monoclonal antibody (AS-4C4) was added, and the plate was incubated at room temperature for $30 \mathrm{~min}$. Then, after washing, $100 \mu \mathrm{L}$ of HRP-conjugated anti-mouse IgG was added to each well and incubated at room temperature for $30 \mathrm{~min}$. Finally, $100 \mu \mathrm{L}$ of stop solution was added to each well, and absorbance was measured at $450 \mathrm{~nm}$ using a spectrophotometric plate reader.

\section{ATPlite luminescence assay}

Cells were seeded in a 96-well plate at $5 \times 10^{3}$ cells/well for $24 \mathrm{~h}$ and then treated with $25 \mathrm{nM}$ ouabain as indicated. The cells were lysed, and ATP fluorescence was measured by following the ATPlite Luminescence Assay System manufacturer's instructions. The relative ATP level was quantified as the fluorescence $\times 100 \%$ in the experimental group and normalized to that of the control group.

Mitochondrial metabolism assay

Cells were seeded in Seahorse XFp cell culture microplates at $8 \times 10^{3}$ cells/well for $24 \mathrm{~h}$ and then treated with ouabain as indicated for another $24 \mathrm{~h}$. The culture medium was replaced with seahorse assay medium, and cells were incubated at $37^{\circ} \mathrm{C}$ in a $\mathrm{CO}_{2}$-free incubator for $1 \mathrm{~h}$. The assay medium was prepared by adding $100 \mathrm{mM}$ sodium pyruvate, $200 \mathrm{mM}$ L-glutamine and $2.5 \mathrm{M}$ glucose to the Agilent Seahorse XF Base Medium. The seahorse assay was run in an XFp Analyzer System (Agilent Technologies) following the manual provided by the manufacturer. To assess mitochondrial metabolic function, oligomycin $(1.0 \mu \mathrm{M})$, carbonyl cyanide- $p$-(trifluoromethoxy) phenylhydrazone $(\mathrm{FCCP}, 1.0 \mu \mathrm{M})$, antimycin, and rotenone $(0.5 \mu \mathrm{M})$ were sequentially injected, and the measurements were recorded after each injection. After measuring the baseline oxygen consumption rate $(\mathrm{OCR})$, the reduction in OCR following oligomycin injection correlates with mitochondrial respiration associated with cellular ATP production. The FCCP-stimulated OCR was used to measure spare respiratory capacity, i.e., the difference between maximal respiration and basal respiration. Rotenone and antimycin $\mathrm{A}$ were used to inhibit electron transfer chain (ETC) complexes I and III, respectively, to shut down mitochondrial respiration.

\section{Glycolysis assay}

Cells were seeded in Seahorse XFp cell culture microplates and treated with ouabain as stated above. The assay medium was prepared by adding $200 \mathrm{mM}$ L-glutamine to the Agilent Seahorse XF Base Medium. The seahorse assay was run in an XFp Analyzer System (Agilent Technologies) following the manufacturer's instructions. To assess the glycolytic function, glucose $(10 \mathrm{mM})$, oligomycin $(1.0 \mu \mathrm{M})$, and 2-deoxyglucose (2-DG, $50 \mu \mathrm{M})$ were sequentially injected, and the measurements were recorded after each injection. After measuring the extracellular acidification rate (ECAR), the increase in ECAR following glucose correlates with the rate of glycolysis under basal conditions. Oligomycin was used to inhibit mitochondrial ATP production and shift energy production to glycolysis. The increase in ECAR following oligomycin reveals the maximal cellular glycolytic capacity. 2-DG was used to inhibit glycolysis through competitive binding to glucose hexokinase. The decrease in ECAR following 2-DG confirms that the ECAR produced is due to glycolysis.

Measurement of glucose consumption

Cells were seeded in six-well plates at $5 \times 10^{5}$ cells/well, allowed to attach overnight and then incubated with ouabain as indicated for $24 \mathrm{~h}$. The culture medium was collected for the measurement of glucose concentrations, and the optical density value of each sample was individually measured using a glucose assay kit at 490 $\mathrm{nm}$ according to the manufacturer's instructions.

Measurement of lactate production

Cells were seeded in six-well plates at $5 \times 10^{5}$ cells/well and treated with ouabain as indicated for $24 \mathrm{~h}$. The cells were then collected 
a

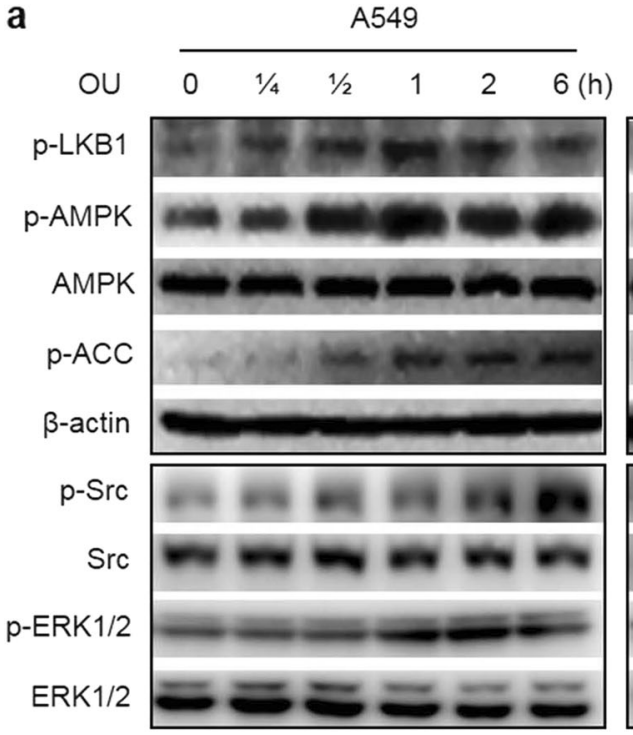

b

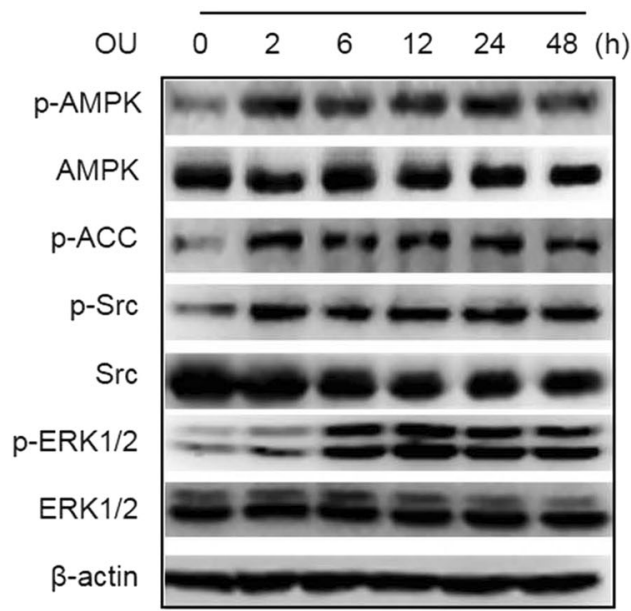

Fig. 1 Activation of AMPK and the Src signaling pathway following time-dependent treatment with ouabain (OU) in A549 and MCF7 cells. The cells were treated with $25 \mathrm{nM}$ ouabain in the short term (a) and long term (b) and then subjected to IB analysis using the respective antibodies, as described in the "Materials and methods" section

and lysed for the detection of lactate production using a lactate assay kit according to the manufacturer's instructions. All values were normalized to the total protein concentration per well [13].

\section{Statistical analysis}

All experiments were repeated at least three times. Statistical analyses were performed using Student's $t$ test (between two groups) or one-way ANOVA (within multiple groups) for data comparisons. For IB analysis, one representative result from at least three experiments is shown. Statistical analyses were performed using SPSS 19.0, and differences between the groups were identified as statistically significant at three levels: $P<0.05$, $P<0.01$, and $P<0.001$.

\section{RESULTS}

Both AMPK and Src signaling pathways are rapidly activated by ouabain in human lung cancer and breast cancer cells

Our work has separately demonstrated the activation of AMPK and Src/ERK $1 / 2$ signaling pathways within $24 \mathrm{~h}$ and $6 \mathrm{~h}$ of ouabain treatment, respectively, in human lung cancer cells $[6,8]$.
However, whether both pathways are simultaneously activated and whether signal activation is present in other cancer tissue cells remain to be examined. The activation of both pathways was checked by IB analysis within $6 \mathrm{~h}$ in A549 lung cancer and MCF7 breast cancer cells after treatment with $25 \mathrm{nM}$ ouabain. The concentration of ouabain falls within the $\mathrm{IC}_{50}$ range in either cell line $[6,14]$. Fig. 1a shows that the simultaneous activation of the AMPK and Src signaling pathways was observed as early as $15 \mathrm{~min}$ after treatment, as evidenced by the increase in p-AMPK and its downstream target p-acetyl-CoA carboxylase (p-ACC) [15], as well as the increased level of $p$-Src and its downstream target p-ERK1/ 2. We also examined liver kinase B1 (LKB1) activity, which activates AMPK by phosphorylating Thr172 of the catalytic a-subunit of AMPK [16], and found that the p-LKB1 level was also increased (Fig. 1a). Moreover, increased exposure $(48 \mathrm{~h})$ to ouabain confirmed the sustained activation of both signaling pathways in MCF7 cells (Fig. 1b). This finding indicates that both AMPK- and Src-dependent signaling pathways are activated by ouabain.

Consistently, results from the in vitro AMPK kinase assay also indicated the upregulation of AMPK kinase activity following timedependent treatment with $25 \mathrm{nM}$ ouabain from 1 to $48 \mathrm{~h}$, with 
a

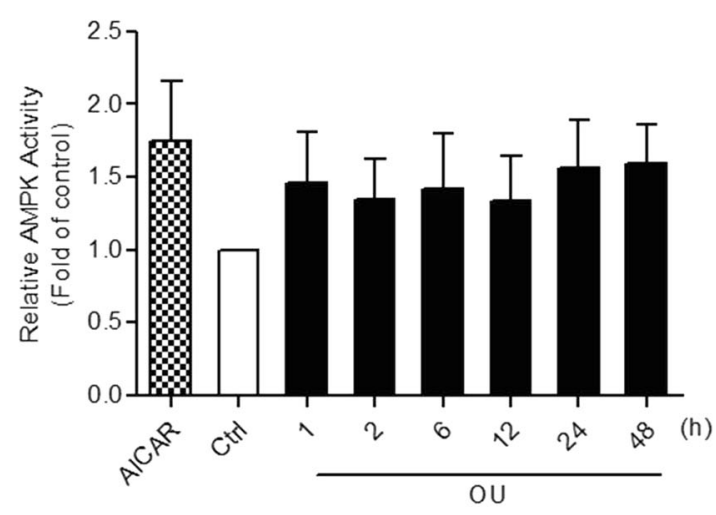

b

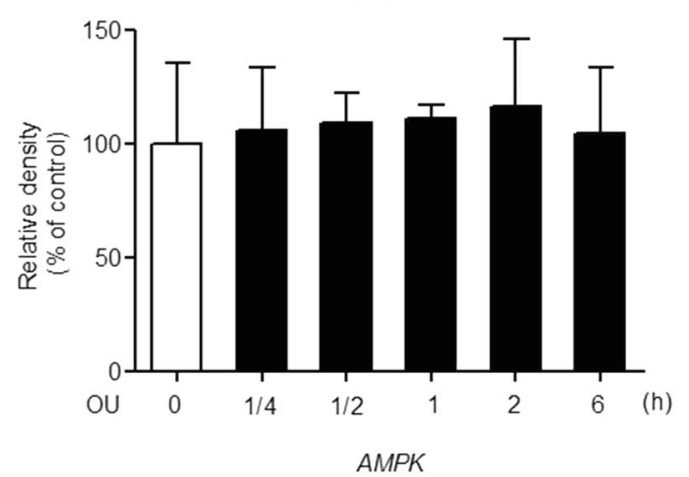

MCF7

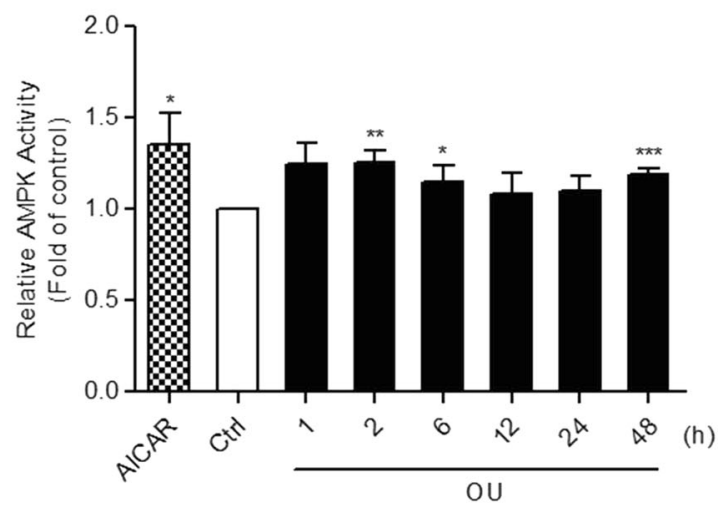

MCF7

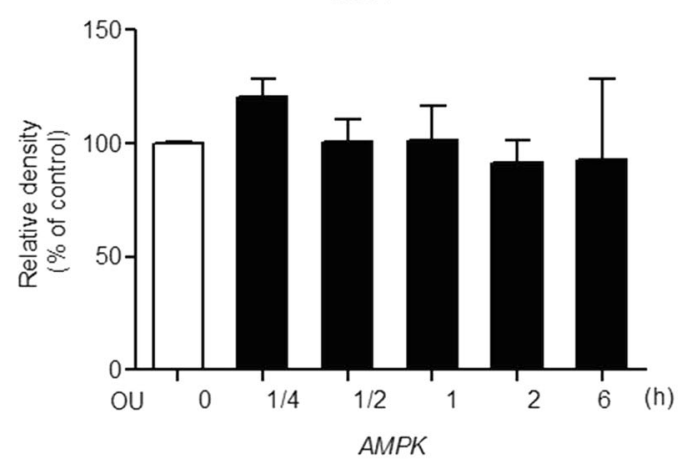

Fig. 2 Effect of ouabain on the activity and the mRNA level of AMPK. The cells were treated with $25 \mathrm{nM}$ ouabain for the indicated time. a The cells were subjected to an AMPK kinase assay, as described in the "Materials and methods" section. AICAR (0.5 mM) treatment for half an hour was used as a positive control. Data are expressed as the mean \pm S.D. of four independent experiments. b Semiquantitative RT-PCR analysis was performed after treatment with ouabain. The intensity of the bands was scanned by ImageJ. Data are expressed as the mean \pm S.D. of three independent experiments. ${ }^{*} P<0.05 ;{ }^{* *} P<0.01 ;{ }^{* *} P<0.001$ versus control

AMPK agonist AICAR serving as a positive control (Fig. 2a), as it can mimic the effect of AMP on AMPK [17]. Further quantitative RTPCR analysis indicated that ouabain affected AMPK activity at the posttranscriptional level, as the compound had little effect on AMPK gene expression in both cell types (Fig. 2b).

AMPK serves as an upstream regulator of Src kinase activation in cancer cells

Although both the AMPK and Src signaling pathways were activated at the same time in cancer cells, whether both pathways regulate each other remains unknown. Thus, the specific AMPK kinase inhibitor compound $C(10 \mu \mathrm{M})$, and the Src kinase inhibitor PP2 $(10 \mu \mathrm{M})$ were used to block the activation of AMPK and Src, respectively, to examine their respective effects. As shown in Fig. $3 a$, compound $C$ treatment expectedly blocked the activation of AMPK in A549 cells after exposure to $25 \mathrm{nM}$ ouabain for $6 \mathrm{~h}$. Interestingly, Src activation was also markedly blocked by compound $\mathrm{C}$ treatment. In contrast, PP2, along with ouabain treatment, blocked Src activation but had little effect on AMPK signaling. Similar effects were observed in MCF7 cells (Fig. 3a). Consistently, AMPK knockdown with two siRNAs significantly inhibited Src activation (Fig. 3b). These results indicate that AMPK functions upstream of Src kinase signaling.

We further tested whether the regulation of Src by AMPK activity occurs following treatment with other AMPK activators, such as AICAR. Fig. 3c shows that AMPK signaling was quickly activated within $1 \mathrm{~h}$ following $0.5 \mathrm{mM}$ AICAR treatment in A549 cells. Similarly, Src was activated simultaneously, and inhibition of
AMPK activation by compound C significantly inhibited SrC activation upon AICAR treatment, while PP2 treatment had little effect on AMPK activation (Fig. 3c). Similar results were observed in MCF7 cells (data not shown). These data suggest that AMPK, upon activation by either ouabain or AICAR, acts upstream of the Src signaling pathway in cancer cells.

Ouabain-induced ATP depletion activates AMPK

AMPK is a well-known energy sensor involved in maintaining energy homeostasis in response to metabolic stress, including the increase in AMP/ATP ratio during nutrient deficiency [16]. We next checked whether AMPK activation correlates with changes in ATP. As shown in Fig. 4a, intracellular ATP levels in both A549 and MCF7 cells were markedly decreased in a time-dependent manner at $24 \mathrm{~h}$ following $25 \mathrm{nM}$ ouabain treatment. Furthermore, the addition of ATP $(100 \mu \mathrm{M})$ to the medium blocked AMPK activation, indicating that ouabain-induced AMPK activity is affected by intracellular ATP alterations (Fig. 4b).

Ouabain affects mitochondrial respiration and glycolysis in cancer Dysregulated metabolism is one of the hallmarks of cancer [18]. Both aerobic glycolysis (Warburg effect) and mitochondrial OXPHOS may operate simultaneously to produce energy in cancer cells $[19,20]$. We next checked whether energy depletion results from altered metabolism following ouabain treatment. The effect of ouabain on mitochondrial OXPHOS and glycolysis was tested using the Seahorse analyzer by measuring the OCR and ECAR, respectively [21]. The baseline respiration, ATP production, 
a

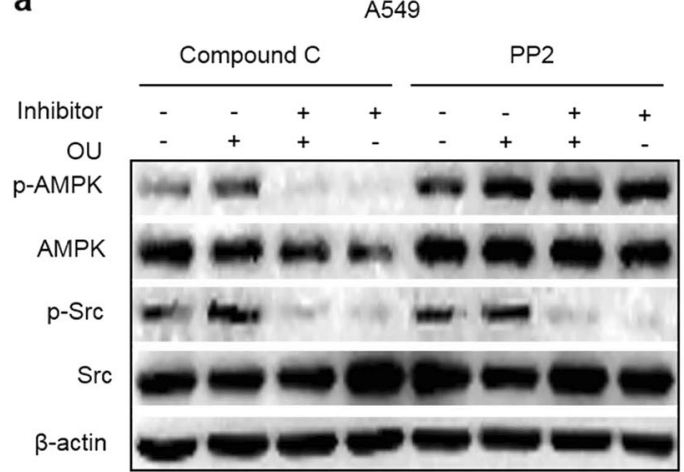

MCF7

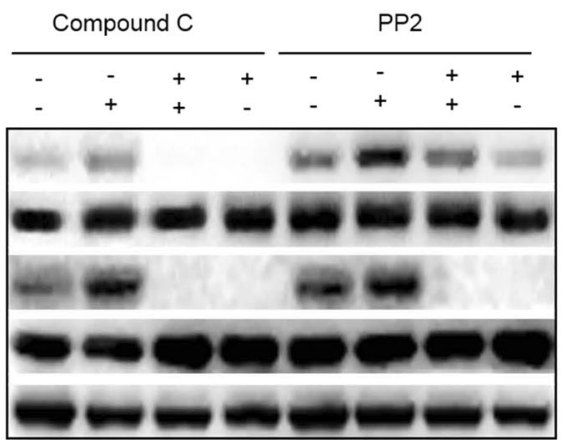

b

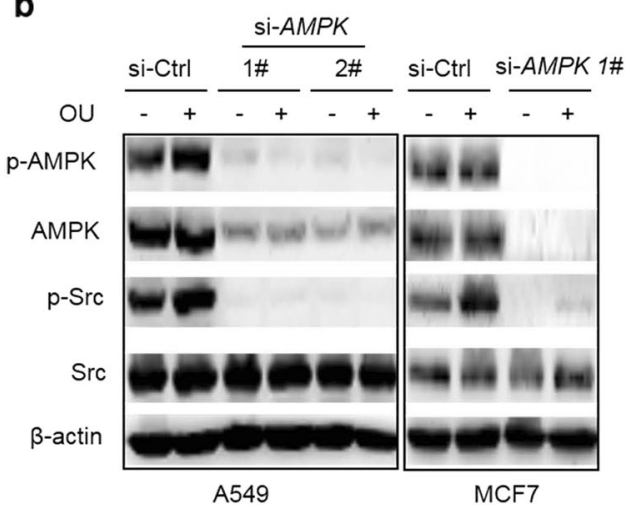

C

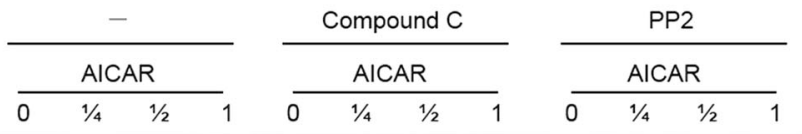

p-AMPK
AMPK
p-Src
Src
$\beta$-actin

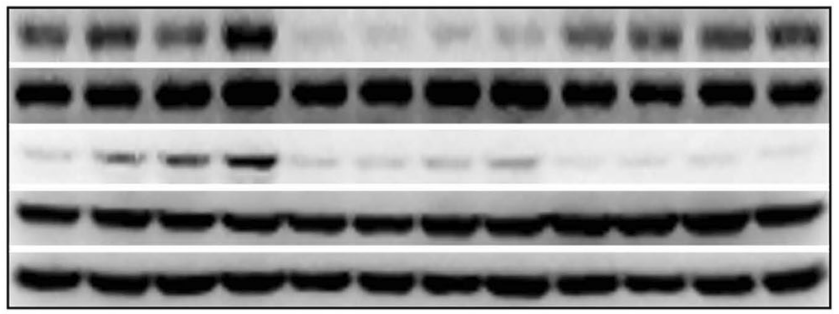

A549

Fig. 3 Effect of specific kinase inhibitors or si-AMPK on ouabain- and AICAR-induced AMPK and Src activation. a A549 and MCF7 cells were treated with $25 \mathrm{nM}$ ouabain in the presence or absence of $10 \mu \mathrm{M}$ compound C or $10 \mu \mathrm{M}$ PP2 for $6 \mathrm{~h}$ and then subjected to IB analysis. $\mathbf{b}$ The cells were transfected with siRNAs targeting AMPK for $48 \mathrm{~h}$ and then treated with $25 \mathrm{nM}$ ouabain for $6 \mathrm{~h}$ and analyzed by IB. c The cells were treated with $0.5 \mathrm{mM}$ AICAR plus $10 \mu \mathrm{M}$ compound C or $10 \mu \mathrm{M}$ PP2 for the indicated time and then subjected to IB analysis

maximum respiration, and spare respiratory capacity are critical parameters demonstrating mitochondrial OXPHOS measured by the Seahorse Mito Stress kit after sequential compound injections. As seen in Fig. 5a, b, the OCR parameters were dose-dependently reduced in A549 cells by ouabain treatment, with a high concentration $(25 \mathrm{nM})$ causing a significant effect on baseline respiration and ATP production. A similar reduction in the OCR was observed in MCF7 cells (Supplementary Fig. 1a, b). This finding indicates that mitochondrial OXPHOS is greatly reduced by ouabain in cancer.

To determine whether AMPK activation occurs before or after OXPHOS reduction, compound $\mathrm{C}$ was added simultaneously with ouabain, and the OCR was subsequently measured. As shown in Fig. $6 a$, cotreatment with compound $C$ did not restore mitochondrial respiration but instead reduced OCR levels, confirming that AMPK was activated following ouabain-reduced OXPHOS.

Furthermore, $25 \mathrm{nM}$ ouabain treatment greatly reduced glycolysis in A549 cells, as demonstrated by the decreased ECAR, as well as by the decreased lactate production and glucose uptake (Fig. 5c-f). However, little effect of the compound was observed on ECAR, and $25 \mathrm{nM}$ ouabain treatment even slightly increased lactate production and glucose uptake in MCF7 cells (Supplementary Fig. 1c-f). We further tested whether the AMPK activator AICAR exerts a differential effect on glycolysis in both cell lines. Interestingly, glycolysis was greatly decreased in A549 cells but slightly increased in MCF7 cells following AICAR treatment, as measured by lactate production and glucose consumption (Fig. 6b, c). This finding indicates that AMPK activation may exert differential effects on glycolysis depending on the cancer cell type.

\section{DISCUSSION}

NKA-targeted therapy has become a valid anticancer strategy since abnormal NKA expression is closely correlated with cancer initiation, progression, and metastasis [22]. In addition to the regulatory role of ion homeostasis played by NKA, the pump is known to function as a receptor that regulates the signal transduction pathway. Ouabain, a well-established NKA inhibitor, has potent anticancer effects in many tissues. Ouabain binds to the a-subunit of NKA and inhibits its ion exchange pump activity. Furthermore, ouabain can initiate the receptor function of NKA, leading to the activation of Src tyrosine kinase and downstream Ras and MAPK (including ERK1/2) cascade activation, as well as various other biological functions [1, 2]. In our previous study, we showed that ouabain activates AMPK and consequently inhibits mTOR activation, leading to autophagic cell death in lung cancer cells, which contribute to the cytotoxicity of the compound [8]. Furthermore, ouabain-induced activation of the Src-ERK1/2 signaling pathway also positively regulates autophagic cell death [6]. In this work, we further demonstrate that (1) AMPK serves as an upstream regulator of Src kinase activation in cancer cells upon ouabain treatment, (2) AMPK activation responds to ouabaininduced ATP depletion, and (3) ouabain greatly reduces mitochondrial respiration while exerting differential cancer cell typedependent effects on glycolysis.

AMPK is a heterotrimer consisting of a catalytic a-subunit and regulatory $\beta$ - and $\gamma$-subunits; the phosphorylation of AMPKa Thr172 is required for the activation of the complex [12]. AMPK plays a pivotal role in many metabolic stress responses and possesses dual tumor suppressing and promoting functions in cancer [23]. In this work, we found that ouabain triggers the rapid 
a

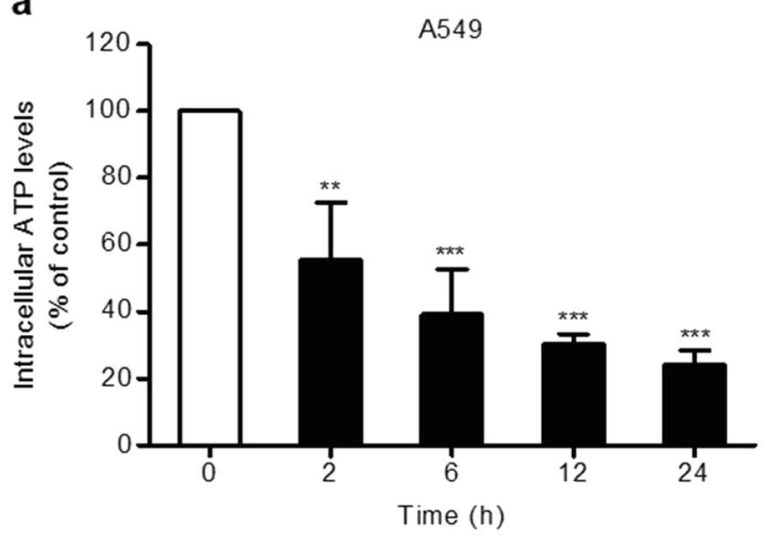

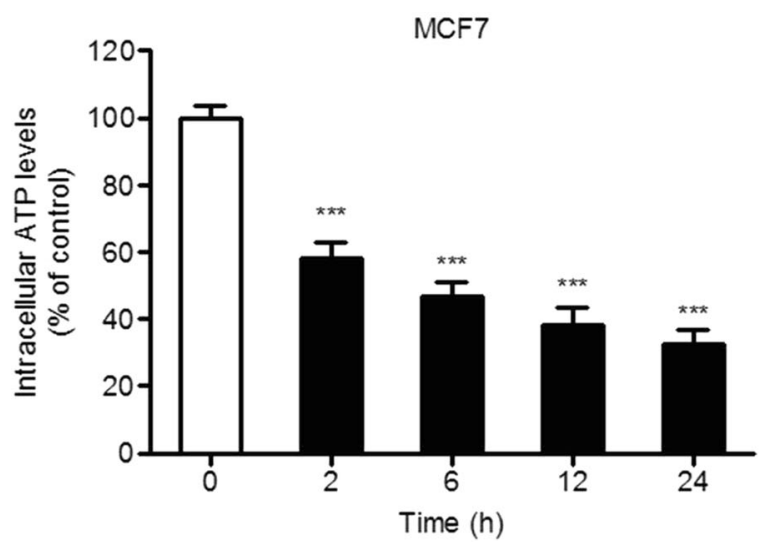

b

ATP

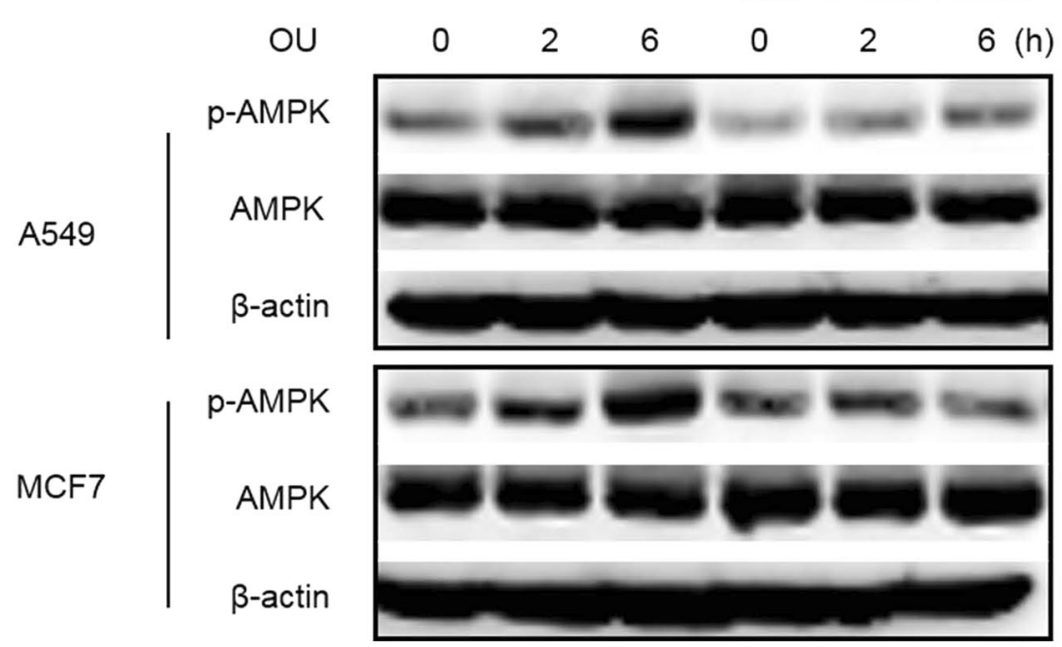

Fig. 4 Ouabain depletes intracellular ATP levels, and the addition of ATP blocks AMPK activation. a Cells were treated with $25 \mathrm{nM}$ ouabain for the indicated time, and the ATP levels were measured with the ATPlite Luminescence Assay System as described in the "Materials and methods" section. ${ }^{* *} P<0.01 ;{ }^{* * *} P<0.001$ versus control. $\mathbf{b}$ The cells were treated with ouabain in the presence or absence of ATP (100 $\left.\mu \mathrm{M}\right)$ for the indicated time and subjected to IB analysis

activation of AMPK activity as early as $15 \mathrm{~min}$, which is much earlier than the $2 \mathrm{~h}$ timeframe in our previous study [8]. The enhanced AMPK activity is evidenced by the increase in phosphorylation of AMPKa Thr172 and the increase in phosphorylation of ACC, a direct AMPK target protein [15], by IB analysis. AMPK activity was also confirmed in the in vitro kinase assay. Given that LKB1 is one of the primary kinases that phosphorylates AMPK [24], our data also confirm the increase in LKB1 activity upon ouabain treatment, indicating that the phosphorylation of AMPK is activated, at least partially, by LKB1.

Although we identified ouabain as an AMPK activator at the nanomolar level in cancer in our present and previous work, the compound was previously reported to inhibit AMPK activity at micromolar doses in rat parotid acinar cells either with or without carbachol stimulation [25]. This discrepancy may result from the large differences with regard to the drug concentration and cellular contexts in the two studies, given that CGs are well known to initiate diverse signaling and biological effects at various concentrations in many cell types. Consistent with our data, other compounds in the CGs family, such as proscillaridin A, bufalin, and digitoxin, have been reported to induce AMPK activation at the nanomolar level in either cancer cell lines or normal human foreskin fibroblast cells, contributing to apoptosis or autophagy [26-29]. Interestingly, AMPK activation by proscillaridin A has been suggested to be correlated with the elevation of the intracellular $\mathrm{Ca}^{2+}$ level, as the interruption of the NKA pump activity by CGs normally stimulates $\mathrm{Na}^{+} / \mathrm{Ca}^{2+}$ exchange, leading to an increase in the calcium ion level [26]. The increased $\mathrm{Ca}^{2+}$ concentration can consequently stimulate calmodulin-dependent kinase kinase $\beta$, which is another positive upstream regulator of AMPK [12]. Although the intracellular $\mathrm{Ca}^{2+}$ concentration was not assessed in the present work, the role of $\mathrm{Ca}^{2+}$ in AMPK activation may not be excluded, given the universal role of ouabain as an NKA inhibitor. Notably, CGs have recently been reported to cause selective anticancer effects in STK11 (LKB1) mutant cells rather than in STK11 wild-type cells, and AMPK activity is quickly inhibited by $100 \mathrm{nM}$ digoxin treatment in A549 lung cancer cells [30]. This work suggests that AMPK activation may provide a resistance mechanism to the effects of CGs, given that functional STK11-AMPK signaling is important for cancer cells to overcome stress responses induced by anticancer agents [31]. These data reflect the complicated responses of AMPK upon CGs treatment in cancer cells under different research conditions.

Here, we report that AMPK functions upstream of the Src signaling pathway upon ouabain or AICAR treatment in A549 and MCF7 cancer cells. Consistent with our findings, metformin has been shown to activate the AMPK-Src-Cbl axis pathway, and compound C blocks Src activation in 3T3-L1 preadipocytes [32]. 
a
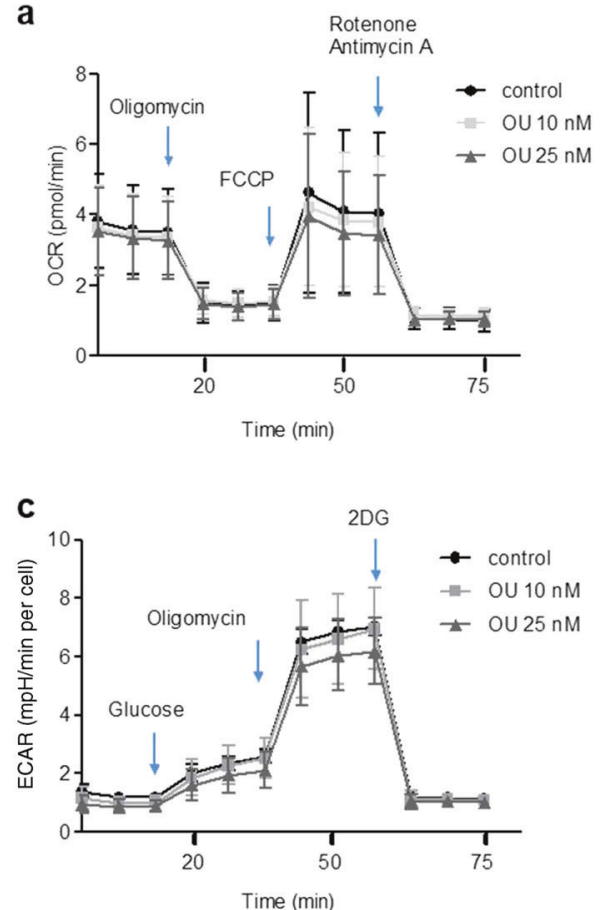

b

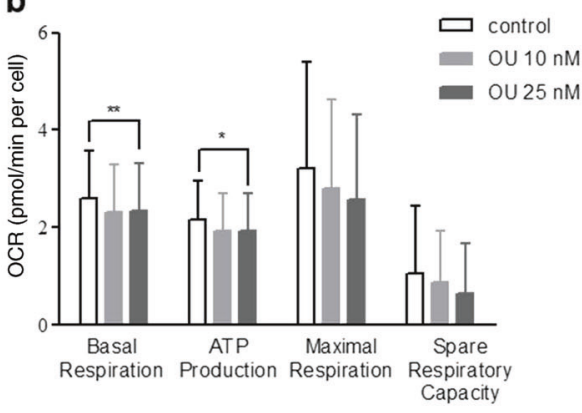

d

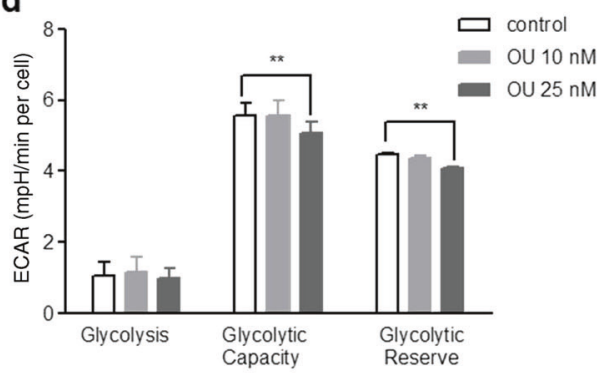

e

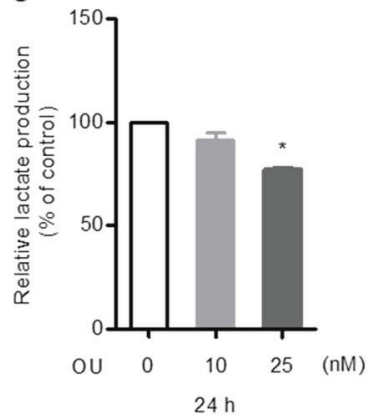

f

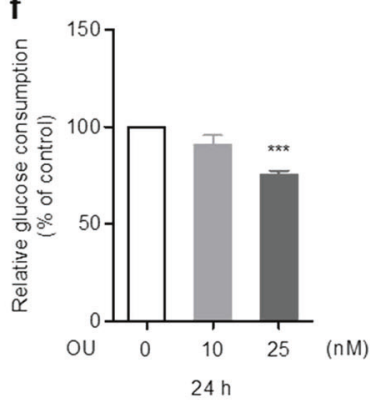

Fig. 5 Regulation of mitochondrial respiration and glycolysis by ouabain in A549 cells. a, b A549 cells were treated with ouabain as indicated for $24 \mathrm{~h}$, and mitochondrial respiration was measured as described in the "Materials and methods" section. ${ }^{*} P<0.05 ;{ }^{* *} P<0.01$. c , d The cells were treated with ouabain as indicated for $24 \mathrm{~h}$, and the glycolytic function of mitochondria was measured as described in the "Materials and methods" section. ${ }^{* *} P<0.01$. e, f A549 cells were treated with ouabain as indicated for $24 \mathrm{~h}$ and analyzed with a lactate assay and glucose assay. ${ }^{*} P<0.05 ;{ }^{* * * P} P<0.001$ versus control
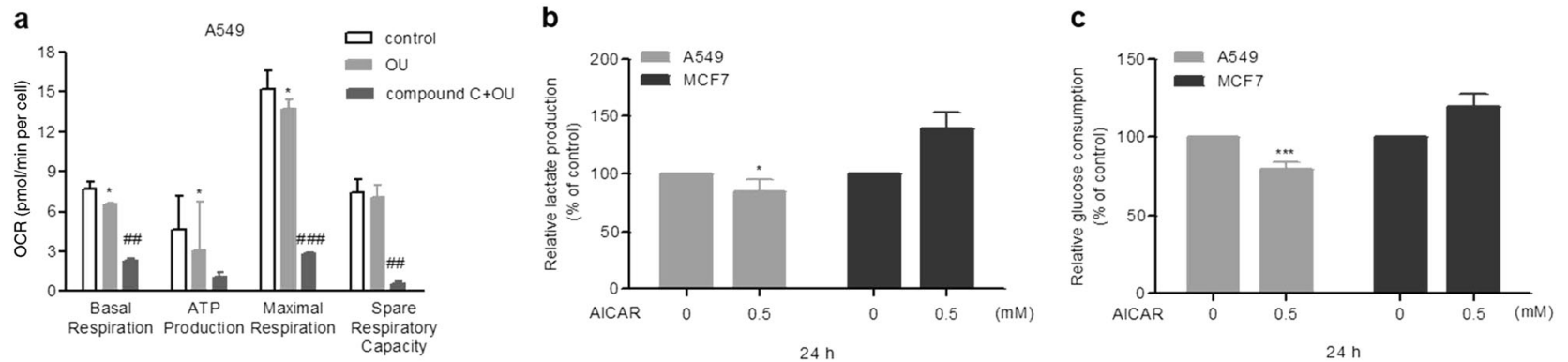

Fig. 6 Effect of compound C and AICAR on mitochondrial respiration and glycolysis. a A549 cells were treated with $25 \mathrm{nM}$ ouabain in the presence or absence of $10 \mu \mathrm{M}$ compound $C$ for $24 \mathrm{~h}$, and mitochondrial respiration was measured. ${ }^{*} P<0.05$ versus control. $\# \#<<0.01$; $\# \# \#<0.001$ versus $25 \mathrm{nM}$ ouabain. b, c A549 and MCF7 cells were treated with $0.5 \mathrm{mM}$ AICAR for $24 \mathrm{~h}$, and assessed by lactate and glucose assays ${ }^{*} P<0.05 ;{ }^{* *} P<0.001$ versus control

Another study also showed that AMPK contributes to SrC activation in endothelial cells or THP-1 monocytes [9, 33]. However, there are reports showing that AMPK activation by either metformin, $\mathrm{ONOO}^{-}$, or estrogen is mediated by Src activation [10, 11, 34]. Furthermore, Src controls AMPK activity by signaling through $\mathrm{PKCa}, \mathrm{PLC} \gamma$, and $\mathrm{LKB} 1$ in certain cancer cell lines, including L-HF1, BP-HF-1, OVCAR3, and A431 [35]. These findings indicate that the cross-regulation between AMPK and Src pathway is complicated and may be cell type specific. Although Src activation is associated with the interaction with the NKA pump following CGs binding [1, 2], and Src interacts with AMPK [32], little is known about the correlation of AMPK with NKA, which is worthy of further research.

Growing evidence indicates that OXPHOS, along with aerobic glycolysis, is a common metabolic phenotype in cancer [20,36]. OXPHOS is an efficient mitochondrial metabolism for the generation of ATP by the tricarboxylic acid cycle. Our findings suggest that ouabain-induced AMPK activation responds to ATP depletion from impaired OXPHOS, based on the fact that timedependent depletion of ATP levels occurs in parallel with the timing of AMPK activation and that supplementation with ATP blocks AMPK activity (Figs. 4 and 5). Consistent with the positive regulatory role of AMPK in OXPHOS through the stimulation of mitochondrial biogenesis [37], cotreatment with the AMPK inhibitor compound $\mathrm{C}$ and ouabain further inhibited OXPHOS (Fig. 6a). The ATP-depleting effect of ouabain confirms our previous observation and indicates that ouabain induces starvation-associated molecular signaling [8]. However, how ouabain impacts metabolism remains elusive. It is possible that ouabain may target ETC complexes to inhibit OXPHOS, much like metformin does [21, 38], although further experiments are required to test this hypothesis.

Aerobic glycolysis is a special metabolism phenotype in cancer [39]. The impairment of OXPHOS by anticancer drugs that activate 
AMPK always boosts glycolysis [21, 40]. However, we found that ouabain treatment decreases glycolysis in A549 cells but has a marginal effect on glycolysis in MCF7 cells. Interestingly, the AMPK activator AICAR similarly induces different metabolic effects on glycolysis in A549 and MCF7 cells. This finding indicates that the AMPK activating-agent-induced metabolic response may vary depending on the cancer cell type. AMPK negatively regulates glycolysis by inhibiting HIF-1a, a key regulator of glycolysis [41]; thus, the inhibition of glycolysis in A549 cells may occur in response to AMPK activation by ouabain. However, the possibility that ouabain directly inhibits glycolysis may not be excluded, as CGs are reported to be capable of inhibiting HIF-1a synthesis [5]. Thus, the complicated metabolic response to ouabain may reflect a combination of the differential metabolic plasticities in various cancer types and the nature of the class of CGs [19].

Taken together, these results demonstrate the detailed regulation between cancer metabolism and AMPK activation by ouabain. The altered cell metabolism may contribute to the cancer cell cytotoxicity of ouabain.

\section{ACKNOWLEDGEMENTS}

This work was supported by grants from the National Natural Science Foundation of China (81872286 and 81621064 to ZW), the CAMS Innovation Fund for Medical Sciences (2016-12M-2-002 to ZW; 2019-12M-1-003 to JS), the CAMS Medical Epigenetics Research Center (2018PT31030 to ZW), and the Drug Innovation Major Project (2018ZX09711001-003-006 to ZW).

\section{AUTHOR CONTRIBUTIONS}

ZW designed the conception and supervised the study. JJS, YCZ, HYL, and ZW developed methodology in the project. JJS, YCZ and HYL performed the experiments. $J J S, H Y L$, and ZW analyzed the data. ZW and JJS wrote, submitted, and revised the manuscript.

\section{ADDITIONAL INFORMATION}

The online version of this article (https://doi.org/10.1038/s41401-019-0290-0) contains supplementary material, which is available to authorized users.

Competing interests: The authors declare no competing interests.

\section{REFERENCES}

1. Prassas I, Diamandis EP. Novel therapeutic applications of cardiac glycosides. Nat Rev Drug Discov. 2008;7:926-35.

2. Schneider NFZ, Cerella C, Simoes CMO, Diederich M. Anticancer and immunogenic properties of cardiac glycosides. Molecules. 2017;22:1932.

3. Wang Z, Zheng M, Li Z, Li R, Jia L, Xiong X, et al. Cardiac glycosides inhibit p53 synthesis by a mechanism relieved by Src or MAPK inhibition. Cancer Res. 2009;69:6556-64.

4. Patel S. Plant-derived cardiac glycosides: role in heart ailments and cancer management. Biomed Pharmacother. 2016;84:1036-41.

5. Zhang H, Qian DZ, Tan YS, Lee K, Gao P, Ren YR, et al. Digoxin and other cardiac glycosides inhibit HIF-1alpha synthesis and block tumor growth. Proc Natl Acad Sci USA. 2008;105:19579-86.

6. Wang Y, Zhan Y, Xu R, Shao R, Jiang J, Wang Z. Src mediates extracellular signalregulated kinase $1 / 2$ activation and autophagic cell death induced by cardiac glycosides in human non-small cell lung cancer cell lines. Mol Carcinog. 2015;54:26-34. Suppl 1E

7. Menger L, Vacchelli E, Adjemian S, Martins I, Ma Y, Shen S, et al. Cardiac glycosides exert anticancer effects by inducing immunogenic cell death. Sci Transl Med. 2012;4:143ra199.

8. Wang Y, Qiu Q, Shen JJ, Li DD, Jiang XJ, Si SY, et al. Cardiac glycosides induce autophagy in human non-small cell lung cancer cells through regulation of dual signaling pathways. Int J Biochem Cell Biol. 2012;44:1813-24.

9. Chang MY, Huang DY, Ho FM, Huang KC, Lin WW. PKC-dependent human monocyte adhesion requires AMPK and Syk activation. PLoS ONE. 2012;7:e40999.
10. Zou MH, Hou XY, Shi CM, Kirkpatick S, Liu F, Goldman MH, et al. Activation of 5'AMP-activated kinase is mediated through c-Src and phosphoinositide 3-kinase activity during hypoxia-reoxygenation of bovine aortic endothelial cells. Role of peroxynitrite. J Biol Chem. 2003;278:34003-10.

11. Zou MH, Kirkpatrick SS, Davis BJ, Nelson JS, Wiles WG, Schlattner U. et al. Activation of the AMP-activated protein kinase by the anti-diabetic drug metformin in vivo. Role of mitochondrial reactive nitrogen species. J Biol Chem. 2004;279:43940-51.

12. Tyszka-Czochara M, Konieczny P, Majka M. Recent advances in the role of AMPactivated protein kinase in metabolic reprogramming of metastatic cancer cells: targeting cellular bioenergetics and biosynthetic pathways for anti-tumor treatment. J Physiol Pharm. 2018;69:337-49.

13. Shi T, Ma Y, Cao L, Zhan S, Xu Y, Fu F, et al. B7-H3 promotes aerobic glycolysis and chemoresistance in colorectal cancer cells by regulating HK2. Cell Death Dis. 2019;10:308.

14. Bielawski K, Winnicka K, Bielawska A. Inhibition of DNA topoisomerases I and II, and growth inhibition of breast cancer MCF-7 cells by ouabain, digoxin and proscillaridin A. Biol Pharm Bull. 2006;29:1493-7.

15. Carling D, Zammit VA, Hardie DG. A common bicyclic protein kinase cascade inactivates the regulatory enzymes of fatty acid and cholesterol biosynthesis. FEBS Lett. 1987;223:217-22.

16. Alexander A, Walker CL. The role of LKB1 and AMPK in cellular responses to stress and damage. FEBS Lett. 2011;585:952-7.

17. Corton JM, Gillespie JG, Hawley SA, Hardie DG. 5-aminoimidazole-4-carboxamide ribonucleoside. A specific method for activating AMP-activated protein kinase in intact cells? Eur J Biochem. 1995;229:558-65.

18. Pavlova NN, Thompson CB. The emerging hallmarks of cancer metabolism. Cell Metab. 2016;23:27-47.

19. Cairns RA, Harris IS, Mak TW. Regulation of cancer cell metabolism. Nat Rev Cancer. 2011;11:85-95.

20. Yu L, Lu M, Jia D, Ma J, Ben-Jacob E, Levine $H$, et al. Modeling the genetic regulation of cancer metabolism: interplay between glycolysis and oxidative phosphorylation. Cancer Res. 2017;77:1564-74.

21. Thakur S, Daley B, Gaskins K, Vasko VV, Boufraqech M, Patel D, et al. Metformin targets mitochondrial glycerophosphate dehydrogenase to control rate of oxidative phosphorylation and growth of thyroid cancerin vitroandin vivo. Clin Cancer Res. 2018;24:4030-43.

22. Mijatovic T, Dufrasne F, Kiss R. $\mathrm{Na}^{+} / \mathrm{K}^{+}$-ATPase and cancer. Pharm Pat Anal. 2012;1:91-106.

23. Chuang HC, Chou CC, Kulp SK, Chen CS. AMPK as a potential anticancer targetfriend or foe? Curr Pharm Des. 2014;20:2607-18.

24. Hawley SA, Boudeau J, Reid JL, Mustard KJ, Udd L, Makela TP, et al. Complexes between the LKB1 tumor suppressor, STRAD alpha/beta and MO25 alpha/beta are upstream kinases in the AMP-activated protein kinase cascade. J Biol. 2003;2:28.

25. Soltoff SP, Hedden L. Regulation of ERK $1 / 2$ by ouabain and Na-K-ATPasedependent energy utilization and AMPK activation in parotid acinar cells. Am J Physiol Cell Physiol. 2008;295:C590-9.

26. Li RZ, Fan XX, Duan FG, Jiang ZB, Pan HD, Luo LX, et al. Proscillaridin A induces apoptosis and suppresses non-small-cell lung cancer tumor growth via calciuminduced DR4 upregulation. Cell Death Dis. 2018;9:696.

27. Shen S, Zhang Y, Wang Z, Liu R, Gong X. Bufalin induces the interplay between apoptosis and autophagy in glioma cells through endoplasmic reticulum stress. Int J Biol Sci. 2014;10:212-24.

28. Zhang YZ, Chen X, Fan XX, He JX, Huang J, Xiao DK, et al. Compound library screening identified cardiac glycoside digitoxin as an effective growth inhibitor of gefitinib-resistant non-small cell lung cancer via downregulation of alpha-tubulin and inhibition of microtubule formation. Molecules. 2016;21:374.

29. Mukhopadhyay R, Venkatadri R, Katsnelson J, Arav-Boger R. Digitoxin suppresses human cytomegalovirus replication via $\mathrm{Na}^{+}, \mathrm{K}^{+}$/ATPase alpha1 subunitdependent AMP-activated protein kinase and autophagy activation. J Virol. 2018;92:e01861-17.

30. Kim N, Yim HY, He N, Lee CJ, Kim JH, Choi JS, et al. Cardiac glycosides display selective efficacy for STK11 mutant lung cancer. Sci Rep. 2016;6:29721.

31. Shackelford DB, Abt E, Gerken L, Vasquez DS, Seki A, Leblanc M, et al. LKB1 inactivation dictates therapeutic response of non-small cell lung cancer to the metabolism drug phenformin. Cancer Cell. 2013;23:143-58.

32. Lee JO, Lee SK, Kim JH, Kim N, You GY, Moon JW, et al. Metformin regulates glucose transporter 4 (GLUT4) translocation through AMP-activated protein kinase (AMPK)-mediated Cbl/CAP signaling in 3T3-L1 preadipocyte cells. J Biol Chem. 2012;287:44121-9.

33. Ning WH, Zhao K. Propionyl-L-carnitine induces eNOS activation and nitric oxide synthesis in endothelial cells via $\mathrm{PI} 3$ and Akt kinases. Vascul Pharmacol. 2013;59:76-82. 
34. Fan P, Griffith OL, Agboke FA, Anur P, Zou X, McDaniel RE, et al. c-Src modulates estrogen-induced stress and apoptosis in estrogen-deprived breast cancer cells. Cancer Res. 2013;73:4510-20.

35. Mizrachy-Schwartz S, Cohen N, Klein S, Kravchenko-Balasha N, Levitzki A. Up regulation of AMP-activated protein kinase in cancer cell lines is mediated through c-Src activation. J Biol Chem. 2011;286:15268-77.

36. Wilde L, Roche $M$, Domingo-Vidal $M$, Tanson $K$, Philp N, Curry J, et al. Metabolic coupling and the reverse Warburg effect in cancer: implications for novel biomarker and anticancer agent development. Semin Oncol. 2017;44:198-203.

37. Chaube B, Malvi P, Singh SV, Mohammad N, Viollet B, Bhat MK. AMPK maintains energy homeostasis and survival in cancer cells via regulating p38/PGC-1amediated mitochondrial biogenesis. Cell Death Discov. 2015;1:15063.
38. El-Mir MY, Nogueira V, Fontaine E, Averet N, Rigoulet M, Leverve X. Dimethylbiguanide inhibits cell respiration via an indirect effect targeted on the respiratory chain complex I. J Biol Chem. 2000;275:223-8.

39. Vander Heiden MG, Cantley LC, Thompson CB. Understanding the Warburg effect: the metabolic requirements of cell proliferation. Science. 2009;324:1029-33.

40. Daurio NA, Tuttle SW, Worth AJ, Song EY, Davis JM, Snyder NW, et al. AMPK activation and metabolic reprogramming by tamoxifen through estrogen receptor-independent mechanisms suggests new uses for this therapeutic modality in cancer treatment. Cancer Res. 2016;76:3295-306.

41. Faubert B, Boily G, Izreig S, Griss T, Samborska B, Dong Z, et al. AMPK is a negative regulator of the Warburg effect and suppresses tumor growth in vivo. Cell Metab. 2013;17:113-24. 